\title{
Serum level of eosinophilic cationic protein in relation to eosinophilia and to exhaled nitric oxide value in children with atopic asthma and aeroallergens sensitization
}

\author{
Ana-Maria Moiceanu Sovarel ${ }^{1,2}$, Eugenia Buzoianu ${ }^{1,3}$, Mariana Moiceanu ${ }^{3}$, \\ Doina Anca Plesca ${ }^{1,3}$ \\ 1"Carol Davila" University of Medicine and Pharmacy, Bucharest, Romania \\ 2"Sf. Pantelimon" Emergency Hospital, Bucharest, Romania \\ 3"Dr. Victor Gomoiu" Clinical Children Hospital, Bucharest, Romania
}

\begin{abstract}
Aim. To assess the correlation between the serum level of eosinophilic cationic protein (S-ECP) and the occurrence of eosinophilia, respectively the exhaled nitric oxide value (FeNO), in children with atopic asthma and aeroallergens sensitization.

Material and method. A prospective study including 63 children with atopic asthma and aeroallergens sensitization aged 5-18 years old, conducted in Pediatric Department of Children Clinical Hospital "Dr. Victor Gomoiu", between April 2016 and July 2017.

The S-ECP level, occurrence of eosinophilia and FeNO value were initially determined. Thereafter the statistically significance of the correlation between S-ECP and the occurrence of eosinophilia, respectively FeNO value, was assessed.

Results. 22 patients had normal S-ECP level; among them only 8 had eosinophilia. 41 patients had increased S-ECP level; among them 28 had eosinophilia. Using the statistical function Pearson Chi-Square Test to assess the significance of the correlation between increased S-ECP level and eosinophilia we have obtained a $\mathrm{p}$ value = 0.0146 (statistically significant).

15 of those 22 patients with normal S-ECP level had at the same time a normal FeNO level; the other 7 patients had increased FeNO level. 17 of those 41 patients with increased S-ECP value had normal FeNO level; the other 24 patients had increased FeNO level. Using the statistical function Pearson Chi-Square Test to assess the significance of the correlation between S-ECP level and FeNO value we have obtained a p value $=0.0432$ (statistically significant).

Conclusion. Increased S-ECP level is correlated with the occurrence of eosinophilia and also with the increased FeNO value in atopic children with aeroallergens sensitization.
\end{abstract}

Keywords: allergic asthma, atopy, eosinophilic cationic protein, eosinophilia, nitric oxide

\section{INTRODUCTION}

Allergic asthma is the most common chronic pathology in the pediatric population.

Asthma is characterized by a high heterogeneity in terms of clinical phenotypes, severity of airway obstruction, degree of obstruction reversibility and response to treatment (1).

The etiology of bronchial asthma is extremely complex and it implies the interaction between multiple types of factors (8). Among these we can mention individual factors (primary factors, most of them genetics) and environmental factors (8).

Among individual factors are included genetic factors (related to atopy, bronchial hyper reactivity, treatment responsivity, bronchial remodeling and disease progression), gender, physical activity and obesity (8).

Over the years several classifications of asthma phenotypes have been proposed based on clinical and pathophysiological parameters, triggers, type of bronchial inflammation, temporal features of the disease etc. (1). 
The most frequent used classification of bronchial asthma in clinical practice is the one based on the presence of atopy: atopic (allergic) and non-atopic phenotype of asthma.

The most common phenotype of asthma in children is atopic (allergic) asthma, being fond in over $80 \%$ of the patients. This phenotype is often associated with a family history of allergic diseases, such as atopic dermatitis, allergic rhinitis, allergic conjunctivitis, urticaria etc. (2).

The athopy represents a genetic particularity involving the individual immune response mediated by immunoglobulins E (IgE) against common environmental aeroallergenes. It is associated with increased IgE level in serum, with antigen - IgE specific response and positive cutaneous tests. We must mention that the production of basal level of $\mathrm{IgE}$ it is under genetic control (8).

Immunoglobulins $\mathrm{E}$ are involved in type I hypersensitivity reactions. They are binding to a specific receptor of the mast cells and basophiles.

The individual exposure to an allergen for which there is allergic sensitization will determine an increase in synthesis of specific IgE and therefore a cascade of allergic-type inflammatory reactions in the bronchial mucosa.

In some cases the presence of atopy is accompanied by some specific clinical allergic manifestations (cough, dyspnea wheezing, thoracic constriction), which are precipitated through the contact with the allergen for which there is allergic sensitization.

In other cases, in the absence of specific clinical manifestations of allergic disease, it is necessary to document atopy using specific tests (3).

Confirmation of sensitization requires investigations, such as skin prick testing or assessment of specific serum immunoglobulin E (IgE).

It is believed that skin prick tests results and specific serum IgE levels may be correlated with clinical manifestations.

The presence of increased serum levels of specific IgE or a positive skin prick test indicates that the individual has been previously exposed to that specific allergen and he is sensitized. Therefore he is at risk to develop allergic-type clinical manifestations when exposed to the allergen on which he is sensitized (7).

The atopic asthma phenotype is typically accompanied by eosinophilic bronchial inflammation.

Children with asthma and aeroallergenic sensitization have often an increased fraction of exhaled nitric oxide (FeNO) and sometimes blood eosinophilia (B-Eos) $(7,8)$.
Both are systemic type (B-Eos), respectively local, bronchial type (FeNO), markers of eosinophilic inflammation (6).

Another marker of systemic eosinophilic inflammation is serum eosinophilic cationic protein (S-ECP), which can be measured in children with asthma (6).

Eosinophilic cationic protein (ECP) is a specific marker, activated and released by eosinophils. ECP triggers histamine release from mast cells, mediating airway hyper reactivity and bronchospasm occurrence (4).

The nitric oxide fraction in exhaled air (FeNO) is the most popular non-invasive biomarker of allergic inflammation in asthma.

The level of exhaled nitric oxide has been shown to be correlated with eosinophilic inflammation of the airway. It may play a role in assessing the degree of asthma control. However it is not routinely used in the monitoring of patients with asthma and $(5,6)$.

\section{CLINICAL STUDY}

The objective of this prospective study was to establish the correlation between:

- the serum cationic eosinophilic protein (S-ECP) as a biomarker of the allergic eosinophilic inflammation and the occurrence of eosinophilia;

- the serum cationic eosinophilic protein (S-ECP) as the biomarker of the allergic eosinophilic inflammation and the exhaled nitric oxide in children with allergic asthma sensitized to aeroallergens.

All the patients included in the study were informed about this and their parents signed the Consent Form approved by the Ethics Commission.

\section{MATERIAL AND METHODS}

It is a prospective study that included 63 children aged between 5 to18 years, which are diagnosed with atopic asthma and sensitized to aeroallergens.

Patients were evaluated in the Pediatrics Department of Clinical Children's Hospital "Dr. Victor Gomoiu" between April 2016 and July 2017.

The inclusion criteria in the study were:

1. age between 5 to 18 years;

2. asthma diagnosis based on anamnestic and clinical data and confirmed using spirometry; a FEV1 reversibility of at least $12 \%$ and/or at least 
$200 \mathrm{ml}$ from baseline after inhaled salbutamol intake was considered positive for asthma diagnosis;

3 . atopy with aeroallergen sensitization documented using increased levels of specific IgE in the pediatric respiratory panel and/or positive skin prick test for at least one aeroallergen (pollen, mites, animal epithelium, mold, insect).

They were excluded from the study:

1. patients with non atopic asthma;

2. patients with other types of sensitization (towards food allergens, medications);

3. patients with atopic asthma which presented comorbidities (gastro esophageal reflux disease, rhinitis, sinusitis).

Prick skin tests were performed with the standard European Stallergenes kit. Skin reactions that occurred after 15 ' were evaluated with the measurement of the papule diameter. Positive reactions have been considered to be those in which the papule had a diameter $\geq 3 \mathrm{~mm}$ compared with the negative control.

Specific serum IgE values were considered positive if they exceeded the level of $0.35 \mathrm{kU} / 1$.

In the first stage of the study for each patient, diagnosed with atopic asthma and aeroallergens sensitization, serum levels of eosinophilic cationic protein (S-ECP) and blood count of eosinophils were determined.
The blood eosinophils count was determined using hemogram (normal eosinophil count was considered $<400$ cells $/ \mathrm{mm}^{3}$ ).

To measure S-ECP the enzyme-linked immunosorbent assay (ELISA) was used. Normal values were considered to be $<13.3 \mu \mathrm{g} / 1$.

For each patient included in the study FeNO was assessed using the NIOX MINO device with a mouthpiece, a chemiluminescence analyzer approved for exhaled NO measurement.

The normal FeNO values were assessed according to age, as depicted in the British asthma management guide from 2014: $<20 \mathrm{ppb}$ in patients $<12$ years and $<25 \mathrm{ppb}$ in patients $\geq 12$ years (7).

Finally the correlation between the S-ECP value and the occurrence of blood eosinophilia, as well as the correlation between the S-ECP value and the FeNO level, were evaluated.

\section{RESULTS}

Among the 63 patients included in the studied group, 22 patients had normal S-ECP value; among them 14 had a normal blood eosinophils count and the other 8 had blood eosinophilia.

41 patients had increased S-ECP value. Out of these, 13 had a normal blood eosinophils count and 28 had blood eosinophilia.

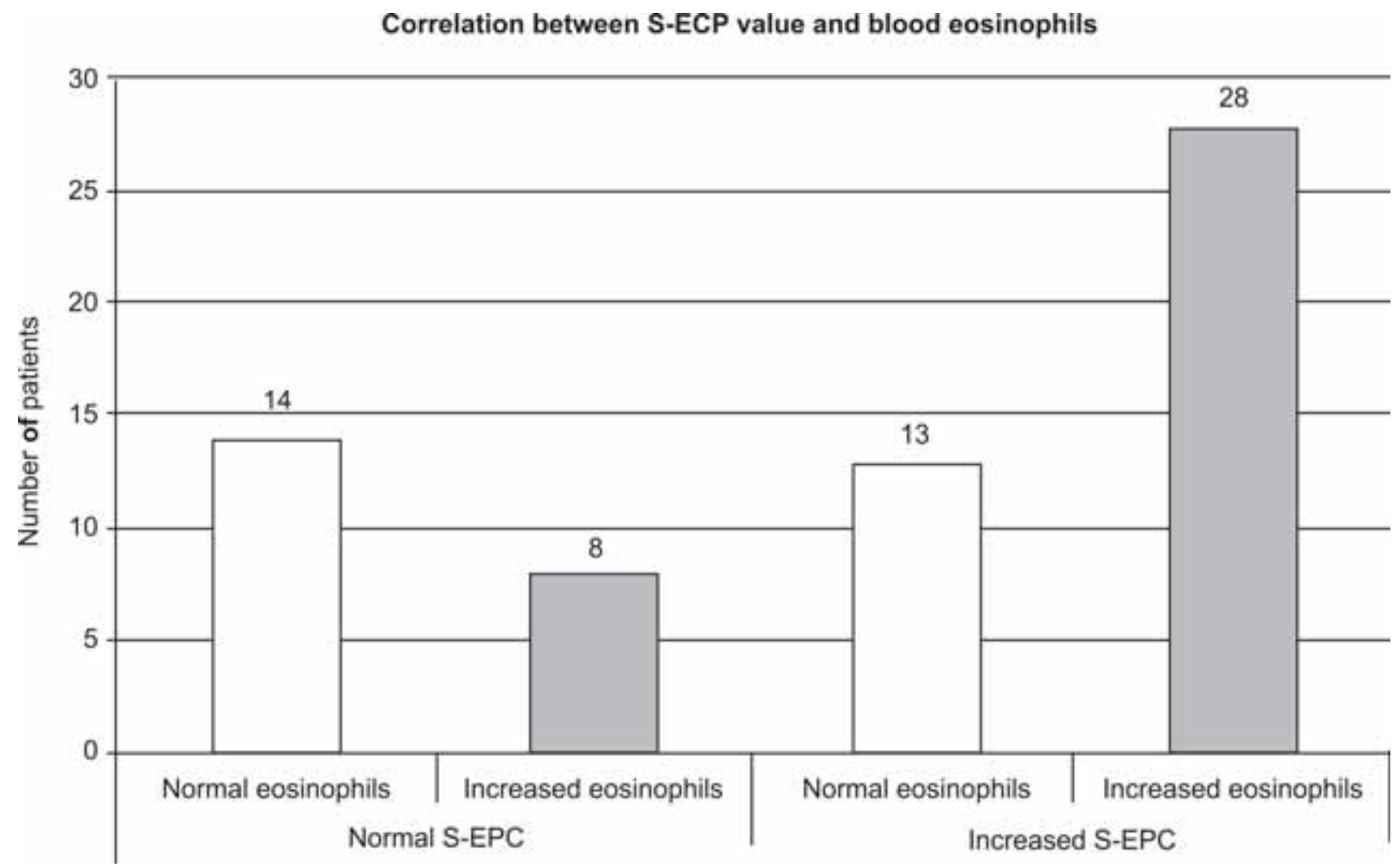

FIGURE 1. Graphic representation of the S-EPC value and blood eosinophils 
In Fig. 1 it is recorded the correlation between the S-ECP level and blood eosinophils.

Using the statistical function Pearson ChiSquare Test to assess the significance of the correlation between increased S-ECP level and blood eosinophilia we have obtained a $\mathrm{p}$ value $=0.0146$ (statistically significant).

15 of the 22 patients with normal S-ECP had a normal FeNO value, while the remaining 7 patients had increased FeNO, as represented in Fig. 2.

17 of the 41 patients with increased S-ECP had normal FeNO values, while the remaining 24 patients had elevated FeNO values, as depicted in the Fig. 2.

In the Fig. 2 it is recorded the correlation between the S-ECP level and FeNO value.

Using the statistical function Pearson ChiSquare Test to assess the significance of the correlation between S-ECP level and FeNO value we have obtained a $\mathrm{p}$ value $=0.0432$ (statistically significant).

\section{DISCUSSIONS}

Specific aeroallergenic sensitization is one of the most important risk factors in development of asthmatic symptoms.
Asthmatic exacerbations determined by allergic triggers occur in $60-90 \%$ of children, and in $50 \%$ of adults.

Measurement of eosinophilic inflammation, resulted from activation of the immune response via Th2 lymphocyte, helps to diagnose, to phenotype and to monitor the disease, in order to detect the deterioration of the asthma control, to identify the patients with risk for exacerbation and to establish the therapeutic attitude (corticosteroid treatment/ biological).

In clinical practice markers of eosinophilic inflammation are often measured using blood, exhaled air and urine. Other methods to identify the inflammation markers (as sputum analysis, aspirate/bronchoalveolar lavage with bronchoscopy) are invasive or difficult to access and can only be performed in ultra specialized centers.

Despite the large variation of inflammation markers described and used in adult clinical studies, only a few of them have been proven useful for asthma management.

Until present an optimal marker for measuring the bronchial inflammation in atopic asthma has yet to be identified.

In the last years many articles concerning the study of inflammation biomarkers in pediatric asth-

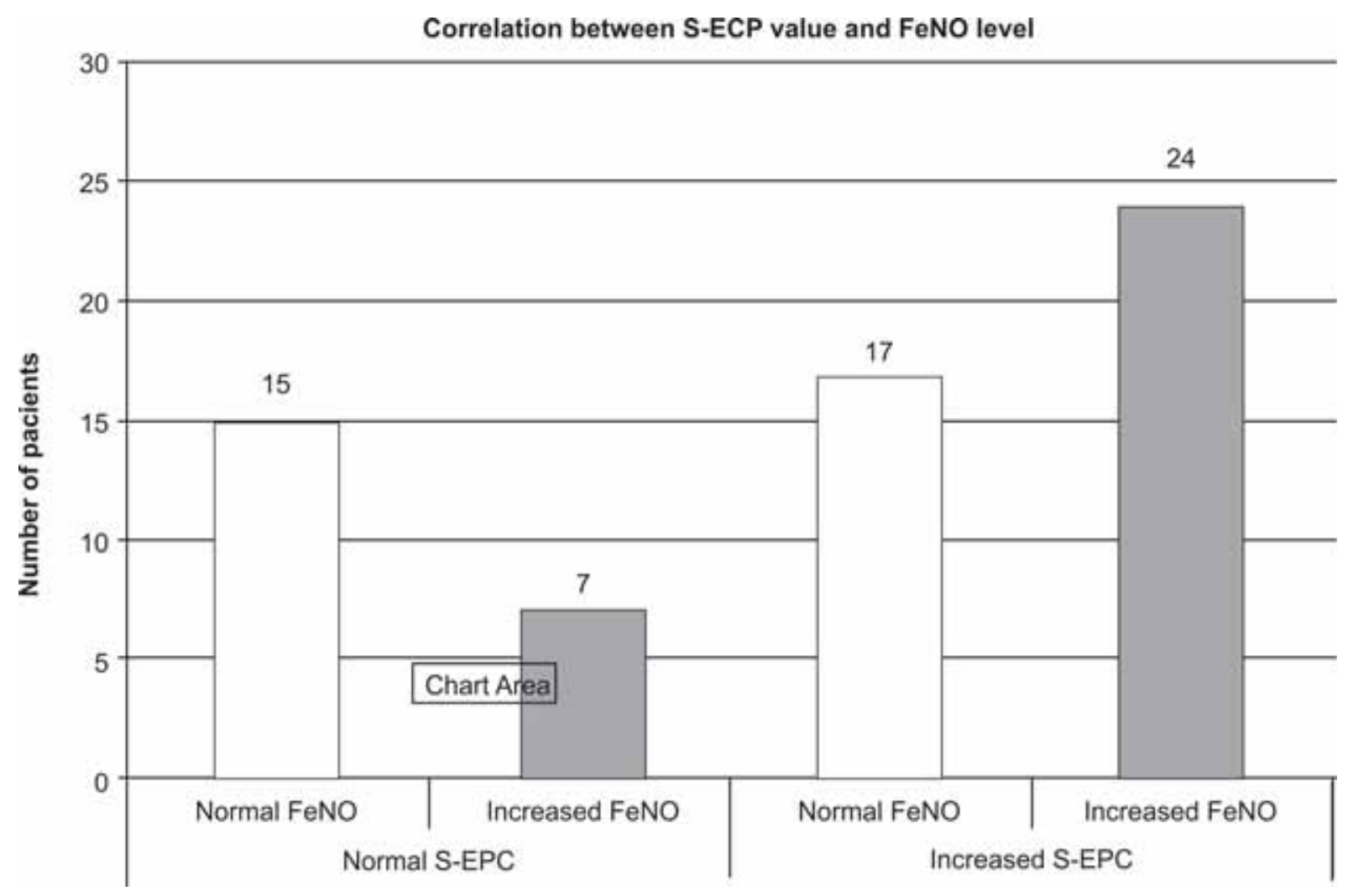

FIGURE 2. Graphic representation of the S-EPC value and FeNO level 
ma have been published, but with controversial results. Current researches attend to identify a marker for the phenotype with increased risk to develop persistent severe asthma to children.

Sometimes the measurement of eosinophilic inflammation alone is not enough and only the mixed evaluation of all these markers, combined with personal history and the pulmonary function helps to diagnose, to phenotype and to appropriate monitor the children with asthma.

\section{CONCLUSIONS}

This study has analyzed if there is any correlation between eosinophilic inflammation markers found in blood and in exhaled air, respectively SCEP level, blood eosinophils and FeNO value.

The prospective study has revealed that:

1. S-ECP value is correlated with blood eosinophils count in children with atopic bronchial asthma sensitized to aeroallergens.

2. S-ECP value is correlated with FeNO value in children with atopic bronchial asthma sensitized to aeroallergens.

3. Assessment of the inflammatory status, using methods that reflect most accurate the bronchial inflammatory process, has practical implications in proper monitoring of the disease evolution under therapy and in preventing of asthma exacerbations.

Conflict of interest: none declared Financial support: none declared

\section{REFERENCES}

1. Zedan M., Attia G., Zedan M.M. et al. Clinical Asthma Phenotypes and Therapeutic Responses, ISRN Pediatrics, 2013; Article ID 824781 http://dx.doi.org/10.1155/2013/824781

2. Global Strategy for Asthma Management and Prevention, Global Initiative for Asthma 2017; http://ginasthma.org/2017-gina-reportglobal-strategy-for-asthma-management-and-prevention

3. Peter J.D., Seamus J.M., Dennis R.B. et al. Roitt's Essential Immunology 13 th edition,city: New York: editure Wiley, 2017: pp 410-418.

4. Morris M.J., Daniel J.P., Zab M. Asthma, Apr 25, 2017; http:// emedicine.medscape.com/article/296301-overview

5. Gross N. Should We Measure FeNO in Asthma? Medscape Pulmonary Medicine, September 16, 2016; http://www.medscape. com/viewarticle/868687
6. Knipping K., Léon M.J.K., Dupont Ch., Garssen J. Serum biomarkers for allergy in children, PAAI 2017; Volume 28: pp: 114-123 http://onlinelibrary.wiley.com/doi/10.1111/pai.12649

7. British guideline on the management of asthma, 2014; 69 Suppl 1: pp 1-192

8. https://www.ncbi.nlm.nih.gov/pubmed

9. Global Strategy for Asthma Management and Prevention, Global Initiative for Asthma 2011; http://ginasthma.org/2011-gina-reportglobal-strategy-for-asthma-management-and-prevention 